\title{
Electronic, phononic, and thermoelectric properties of graphyne sheets
}

\author{
Hâldun Sevinçli ${ }^{1, a)}$ and Cem Sevik ${ }^{2, b)}$ \\ ${ }^{1}$ Department of Materials Science and Engineering, Izmir Institute of Technology, Izmir, TR 35430, Turkey \\ ${ }^{2}$ Department of Mechanical Engineering, Faculty of Engineering, Anadolu University, Eskisehir, TR 26555, \\ Turkey
}

(Received 29 August 2014; accepted 16 November 2014; published online 2 December 2014)

\begin{abstract}
Electron, phonon, and thermoelectric transport properties of $\alpha-, \beta-, \gamma-$, and 6,6,12-graphyne sheets are compared and contrasted with those of graphene. $\alpha-, \beta-$, and 6,6,12-graphynes, with direction dependent Dirac dispersions, have higher electronic transmittance than graphene. $\gamma$-graphyne also attains better electrical conduction than graphene except at its band gap. Vibrationally, graphene conducts heat much more efficiently than graphynes, a behavior beyond an atomic density differences explanation. Seebeck coefficients of the considered Dirac materials are similar but thermoelectric power factors decrease with increasing effective speeds of light. $\gamma$-graphyne yields the highest thermoelectric efficiency with a thermoelectric figure of merit as high as $Z T=0.45$, almost an order of magnitude higher than that of graphene. (C) 2014 AIP Publishing LLC.
\end{abstract}

[http://dx.doi.org/10.1063/1.4902920]

Graphene and its functionalized forms have many extraordinary physical properties, such as robustness, stability, flexibility, very high thermal, and electrical conductivities, ${ }^{1-8}$ which inspired numerous studies exploring the possibilities for adapting them for technological applications in the fields of not only electronics, photonics, and optoelectronics, ${ }^{9-11}$ but also thermoelectrics and thermal management of nano-devices. ${ }^{8,12-18}$ Recently, it was shown that other two-dimensional materials can also be stable and that they widen the potential of two-dimensional materials for applications. ${ }^{19-22}$ Of particular importance are graphene-like carbon allotropes, called graphyne structures composed of one-atom-thick sheets of carbon atoms but containing sp carbon bonds in addition to $\mathrm{sp}^{2}$ hybridized bonds ${ }^{23-25}$ (see Fig. 1). Indeed, assembled subunits of several graphyne structures $^{26-28}$ and another similar graphene allotrope including triple-bonded carbon linkages, so-called graphdiyne films and nanoribbons have already been synthesized. ${ }^{29,30}$ Theoretical calculations have shown that these lowdimensional structures have unique mechanical, ${ }^{24,31-33}$ thermal, ${ }^{34,35}$ optical, ${ }^{36}$ and particularly electrical ${ }^{37-42}$ properties as peculiar as those of graphene. For instance, 6,6,12-graphyne, exhibit direction-dependent conductivity due to its electronic structure possessing two self-doped nonequivalent distorted Dirac cones. ${ }^{38}$ On the other hand, $\gamma$-graphyne has a direct energy band gap, ${ }^{43,44}$ which is appealing for electronic applications. ${ }^{45}$ Considering that one of the most important concerns about graphene is the difficulty to manipulate its electronic conduction due to absence of a band gap, $\gamma$-graphyne was argued to be more suitable for electronics. ${ }^{46}$ More recently, thermoelectric properties of graphynes were reported to have interesting features. ${ }^{35,47,48}$ Yet, the potential of graphynes for applications require further investigations.

In this work, electronic, phononic, and thermoelectric transport properties of four types of graphyne structures, namely, $\alpha$-, $\beta$-, $\gamma$-, and 6,6,12-graphynes, are systematically investigated

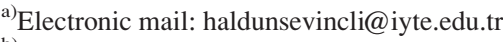

b)Electronic mail: csevik@anadolu.edu.tr
}

from first principles calculations, and they are compared with those of graphene. First principles calculations are performed using the VASP code ${ }^{49,50}$ which is based on density functional theory (DFT). ${ }^{51-53}$ The projector augmented wave pseudopotentials (PAW) $)^{54,55}$ from the standard distribution are incorporated in the calculations. For electronic exchange-correlation functional, the generalized gradient approximation (GGA) in its PBE parametrization ${ }^{56}$ is used. The electronic band structure calculations of all materials are carefully converged by tuning
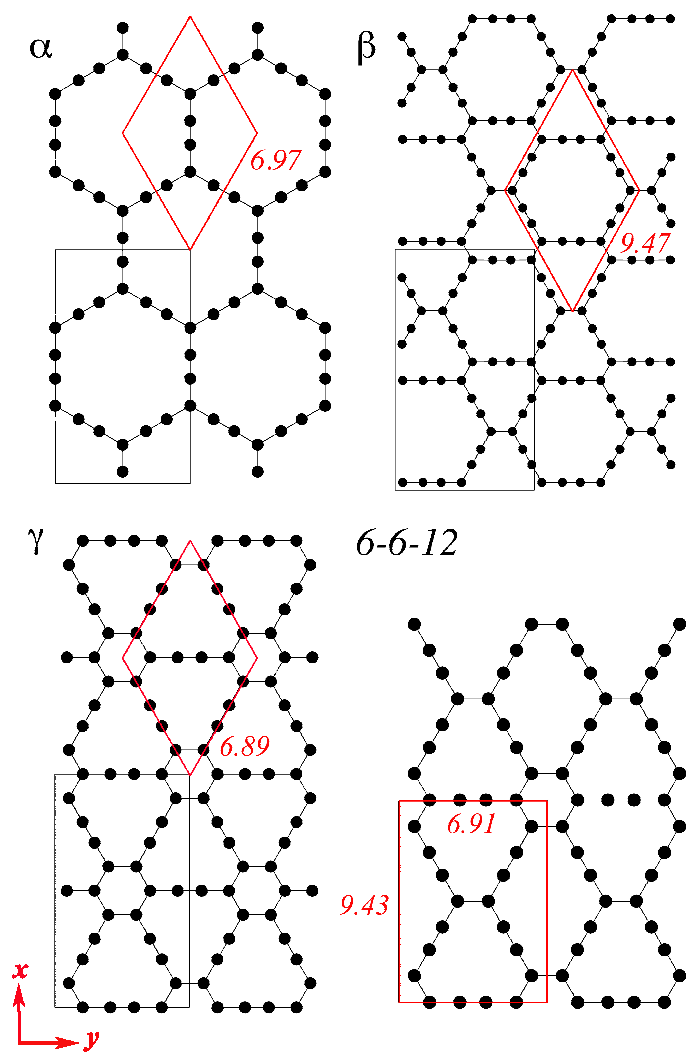

$6-6-12$

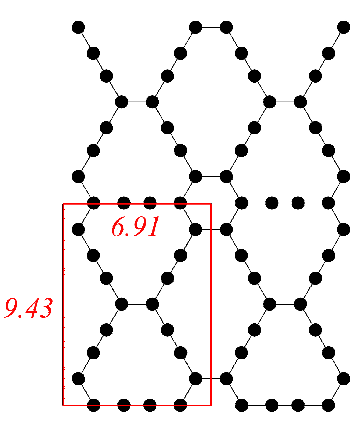

FIG. 1. Schematic representations of $\alpha-, \beta-, \gamma$-, and 6,6,12-graphynes. The red cells represent the corresponding unit cells for each structure and black tetragonal cells represent the conventional cells used in transport calculations. 
the number of Monkhorst-Pack $k$-point grids. ${ }^{57}$ The vibrational frequencies are obtained by using PHONOPY code ${ }^{58}$ which can directly use the force constants calculated by density functional perturbation theory ${ }^{59}$ as implemented in the VASP code. Here, $2 \times 2 \times 1(8 \times 4 \times 1), 2 \times 1 \times 1(6 \times 6 \times 1), 2 \times 2 \times 1$ $(6 \times 4 \times 1)$, and $2 \times 2 \times 1(6 \times 8 \times 1)$, conventional super cell structures ( $\Gamma$ centered $k$-points grids) are considered for $\alpha$-, $\beta$-, $\gamma$-, and 6,6,12-graphyne crystals, respectively. For graphene, $28 \times 28 \times 1 k$-point mesh is used. As the plane wave energy cut off and total energy convergence criteria, $650 \mathrm{eV}$ and $10^{-6} \mathrm{eV}$ are used in all simulations and materials, while the force tolerance is set to $10^{-2} \mathrm{eV} / \AA$.

Transport calculations are performed at the ballistic limit. Vibrational thermal conductance is calculated from $^{60}$

$$
\kappa_{\mathrm{ph}}(T)=\int \frac{d \omega}{2 \pi} \hbar \omega \frac{\partial f_{B}(\omega, T)}{\partial T} \mathcal{T}_{\mathrm{ph}}(\omega),
$$

where $\omega$ is the vibrational frequency, $f_{B}$ stands for the Bose function, and $\mathcal{T}_{\mathrm{ph}}$ is the phonon transmission spectrum. For electrons, we use the functions ${ }^{61}$

$$
L_{n}=-\frac{2}{h} \int d E \frac{\partial f_{F}(E, \mu, T)}{\partial E}(E-\mu)^{n} \mathcal{T}_{\mathrm{el}}(E),
$$

where $n$ is integer, the integral is taken over energy, $\mu$ is the chemical potential, $T$ is temperature, $f_{F}$ is the Fermi function, and $\mathcal{T}_{\text {el }}$ is the electronic transmission spectrum. Using $L_{n}$, one can define the electrical conductance as $G=\mathrm{e}^{2} L_{0}$, Seebeck coefficient as $S=\left(L_{1} / L_{0}\right) / \mathrm{e} T$, and the electrical contribution to thermal conductance as $\kappa_{\mathrm{el}}=\left(L_{2}-L_{1}^{2} / L_{0}\right) / T$. Using these, the power factor is given as $P=S^{2} G$ and the thermoelectric figure of merit is $Z T=S^{2} G T /\left(\kappa_{\mathrm{el}}+\kappa_{\mathrm{ph}}\right)$. In the ballistic limit, the transmission spectra can be obtained from the number of band crossings of a particular energy value. Tetragonal cells depicted in Fig. 1 are considered for transport calculations. In order to obtain accurate results, 200 (100) $k$-points are used in the transverse direction for obtaining electron (phonon) transmission spectra. The optimized first principles lattice constants of $\alpha-\beta$-, and $\gamma$-graphyne are predicted as $6.97 \AA$, $9.47 \AA$, and $6.89 \AA$, respectively. For $6,6,12$-graphyne, they are $9.43 \AA$ and $6.91 \AA$ with $b_{0} / a_{0}=1.3658$, in good agreement with previous works. ${ }^{39,62,63}$

Electronic band structures for $\alpha-, \beta-, \gamma-, 6,6,12$-graphyne, and graphene are depicted in Figs. 2(a)-2(e), respectively. Similar to graphene, the highest valence and the lowest conduction bands of $\alpha, \beta$, and 6,6,12-graphyne structures meet at single $k$-points, named as the Dirac point or the charge
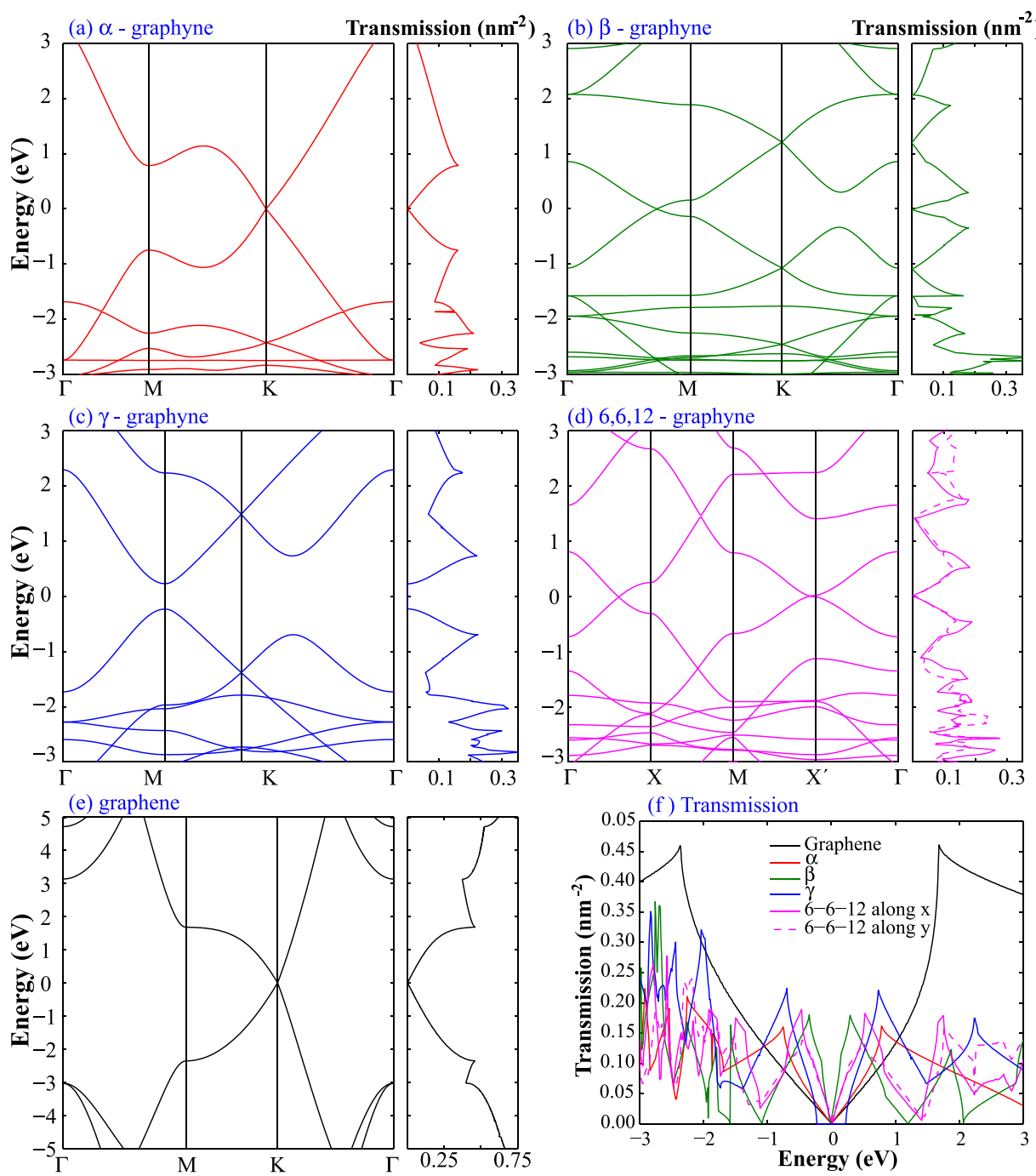

FIG. 2. The calculated electronic band structures of (a) $\alpha$-, (b) $\beta$-, (c) $\gamma$-, (d) 6,6,12-graphyne, and (e) graphene structures, and the corresponding electronic transmissions (f). 
neutrality point $(\mathrm{CNP}), \alpha$-graphyne has two fold symmetric Dirac points at $K$ and $K^{\prime}$ in the Brillouin zone, the same as graphene. However, $\beta$-graphyne has six fold symmetric Dirac points between $\Gamma \leftrightarrow M$ in the Brillouin zone despite its hexagonal lattice symmetry. On the other hand, 6,6,12-graphyne, having tetragonal lattice symmetry, has two different Dirac points with two fold symmetry between $\Gamma \leftrightarrow X$ and $M \leftrightarrow X^{\prime}$ (pretty close to $X^{\prime}$ ). These results are consistent with the previous first principle calculations reported by Malko et al. ${ }^{38}$ Unlike these three graphyne structures, the electronic band structure of $\gamma$-graphyne exhibit semiconducting nature with $\sim 0.5 \mathrm{eV}$ direct energy band gap at $M$ point.

Electronic transmission spectra $\mathcal{T}_{\text {el }}$ per cross section area are also given in Figs. 2(a)-2(e). Note that we use the interlayer spacing of graphite $(3.35 \AA)$ for all structures. In Fig. 2(f), we compare the electronic transmission spectra, where $\kappa_{\mathrm{el}}$ of 6,6,12-graphyne is plotted in two inequivalent directions. Even though the electronic transmittance of graphene is larger than those of graphynes in most of the spectrum, the transmission of $\alpha-, \beta$, and 6,6,12-graphynes are larger than graphene's around the CNP. This is basically due to the slower Fermi velocities, or equivalently higher densities of states around the CNPs of these structures. A comparison of $\mathcal{T}_{\text {el }}$ around CNP with the slopes of the Dirac cones (see Ref. 38) yield a good agreement in the sense that slower Dirac fermions give rise to more rapid rise in transmission values when moving away from the Dirac point. $\gamma$-graphyne, having $0.5 \mathrm{eV}$ band gap and finite effective mass, has considerably large $d \mathcal{T}_{\text {el }} / d E$ value close to the valence and conduction band edges, which is important for thermoelectrics because the Seebeck coefficient is proportional to the logarithmic derivative of $\mathcal{T}_{\text {el }}$ with respect to energy.

Phonon dispersions and phonon transmission spectra of graphynes are plotted in Figs. 3(a)-3(d) and those of graphene in Fig. 3(e). The sp bonds present in graphynes manifest themselves with the appearance of high frequency modes between 60 and $70 \mathrm{THz}$, which are absent in graphene. As is the case for electrons, phonon transmission spectra are slightly different in $x$ - and $y$-directions for 6,6,12-graphyne. When compared to graphene, graphynes' transmission is higher at low frequencies, but graphene has higher transmittance otherwise. An important feature of all graphynes considered in this work is that they have gaps in their phonon bands. These features in phonon dispersions lead to lower thermal conductance of graphynes, compared to graphene for $T \geq 60 \mathrm{~K}$ (Fig. 3(f)).

One can argue that one of the reasons for $\kappa_{\mathrm{ph}} / A$ of graphene being significantly larger than those of graphynes' at room temperature is the fact that graphene atoms are closely packed as opposed to graphynes. We observe that $\alpha$-graphyne (the one with smallest atomic density, $d$ ) has a higher $\kappa_{\mathrm{ph}} / A / d$ value than the other graphynes, but graphene still has larger values for $T>200 \mathrm{~K}$. That is, the atomic density is
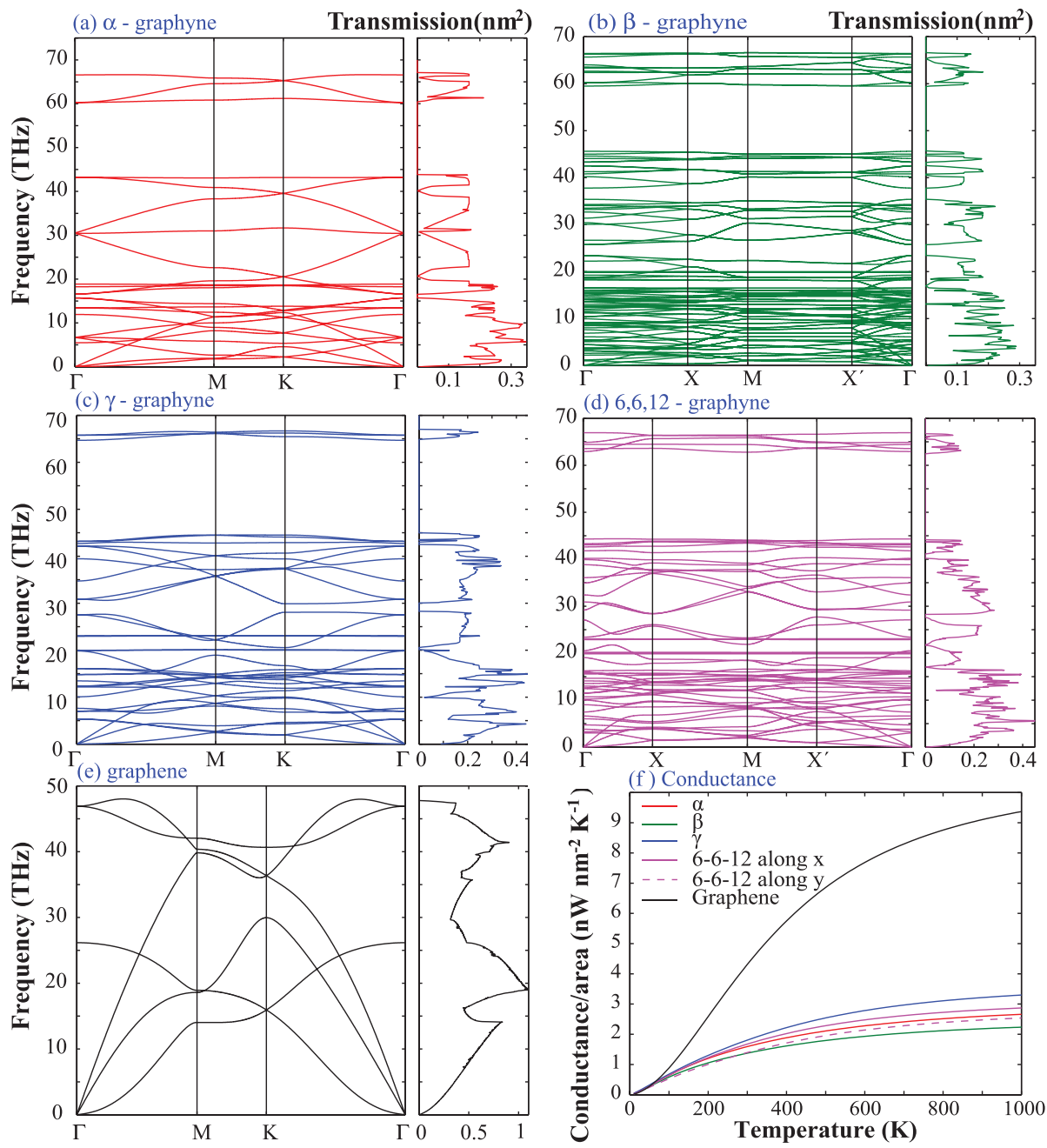

FIG. 3. Phonon dispersions and transmission spectra along $x$-direction. (a) $\alpha$, (b) $\beta$-, (c) $\gamma$-, (d) 6,6,12-graphyne, and (e) graphene. (f) The corresponding thermal conductance values. 
a key factor affecting phononic heat transport but it is not decisive by itself.

Thermoelectric properties of graphynes display interesting features. In Figs. 4(a)-4(e), Seebeck coefficient, power factor, electronic and total thermal conductances, and $Z T$ are plotted as functions of $\mu$ for $T=300 \mathrm{~K}$. $S$ of $\alpha$-, $\beta$-, and 6,6,12-graphynes have similar values and are close to that of graphene (Fig. 4(a)). It is evident from Eq. (2) that the contribution of a Dirac cone to $S$ is independent of the effective speed of light. In the presence of multiple Dirac cones with different slopes and curvatures, if we assume a simple power law dependence of $\mathcal{T}_{\text {el }}$ on $E$, then $S$ should be independent of the power and the prefactor, while $P$ should scale with the same prefactor as $\mathcal{T}_{\text {el }}$. Indeed, at room temperature, the power factors of these structures satisfy $P_{\beta}>P_{6,6,12}>P_{\alpha}>P_{\text {graphene, }}$, as it can be inferred from the transmission spectra at Fig. 2(f). On the other hand, $\gamma$-graphyne has considerably higher $S$, but one should note that its highest values are inside the band gap. In Fig. 4(b), one observes that the maximum $P$ values at room temperature attained for $\gamma$ - and $\beta$-graphynes are at the same order (1.1 and $0.8 \mathrm{pW} \mathrm{nm}^{-2} \mathrm{~K}^{-2}$, respectively), while $6,6,12$ graphyne can yield approximately $0.4, \alpha$-graphyne and graphene have $<0.2 \mathrm{pW} \mathrm{nm}^{-2} \mathrm{~K}^{-2}$.

The detrimental role of thermal conductance on thermoelectric efficiency is apparent in graphynes and graphene based structures. ${ }^{16,64}$ Electronic and total heat conductances at $300 \mathrm{~K}$ are shown in Figs. 4(c) and 4(d), respectively. In the $\pm 0.5 \mathrm{eV}$ energy window, $\beta$ - and 6,6,12-graphynes have higher $\kappa_{\mathrm{el}}$ than the other structures, and $\gamma$ - graphyne yields higher $\kappa_{\mathrm{el}}$ than $\alpha$-graphyne and graphene except at its energy band gap. This order remains the same for $\kappa_{t o t}$ except for graphene. An important feature we observe is that graphene has the lowest $\kappa_{\mathrm{el}}$ in $|\mu|<0.75 \mathrm{eV}$ range among the Dirac materials studied (namely, $\alpha-, \beta-, 6,6,12-$ graphynes, and graphene). But $\kappa_{t o t}$ of graphene is the highest at all $\mu$ values, because of its substantially high $\kappa_{\mathrm{ph}}$ (see Fig. 3 ).

Finally, $Z T$ as a function of the chemical potential at $300 \mathrm{~K}$ and the maximum $Z T$ values at different temperatures $\left(Z T_{\max }\right)$ are plotted in Figs. 4(e) and 4(f), respectively. First, we should note that electron-hole symmetry is preserved for $Z T$ at low energies for all structures we study. At $300 \mathrm{~K}, \beta-$, $6,6,12-$, and $\alpha$-graphynes have their peak $Z T$ values close to their Dirac points with $Z T \simeq 0.12$ for $\beta$-graphyne, 0.05 (0.04) for 6,6,12-graphyne in $x$-(y-)direction, and 0.03 for $\alpha$ graphyne. $\gamma$-graphyne, the only material with a finite band gap in this study, gives rise to the highest $Z T$ value close to its valence and conduction band edges with a maximum value of 0.17 at room temperature. Graphene can support a maximum $Z T$ value of 0.01 at room temperature, lower than all graphynes. This is rooted in the details of both the electronic and the phononic structures. Electronically, even though $S$ of graphene has similar values with those of $\alpha$-, $\beta$-, and 6,6,12-graphynes, $P$ of $\beta$ - and 6, 6, 12-graphynes are significantly larger than that of $\alpha$-graphyne and graphene, because of their lower electrical conductance around the CNP. $\alpha$-graphyne and graphene have similar $P$ and $\kappa_{\mathrm{el}}$ but the superior phonon thermal conductance of graphene is the main reason for its peak $Z T$ value at room temperature being 3 times lower than that of $\alpha$-graphene. $Z T_{\max }$ increases monotonically with temperature for all structures except $\beta$ graphyne, which achieves its peak value at around $500 \mathrm{~K}$. This is mainly because its $S$ and $P$ also change nonmonotonically with temperature. The kink at $800 \mathrm{~K}$ is due to a sudden change of the chemical potential where the maximum takes place, namely, a jump from one band edge to another. $\gamma$-graphyne has the highest values for all temperatures, and the increase is mostly linear with temperature, reaching values as high as 0.45 in the given temperature
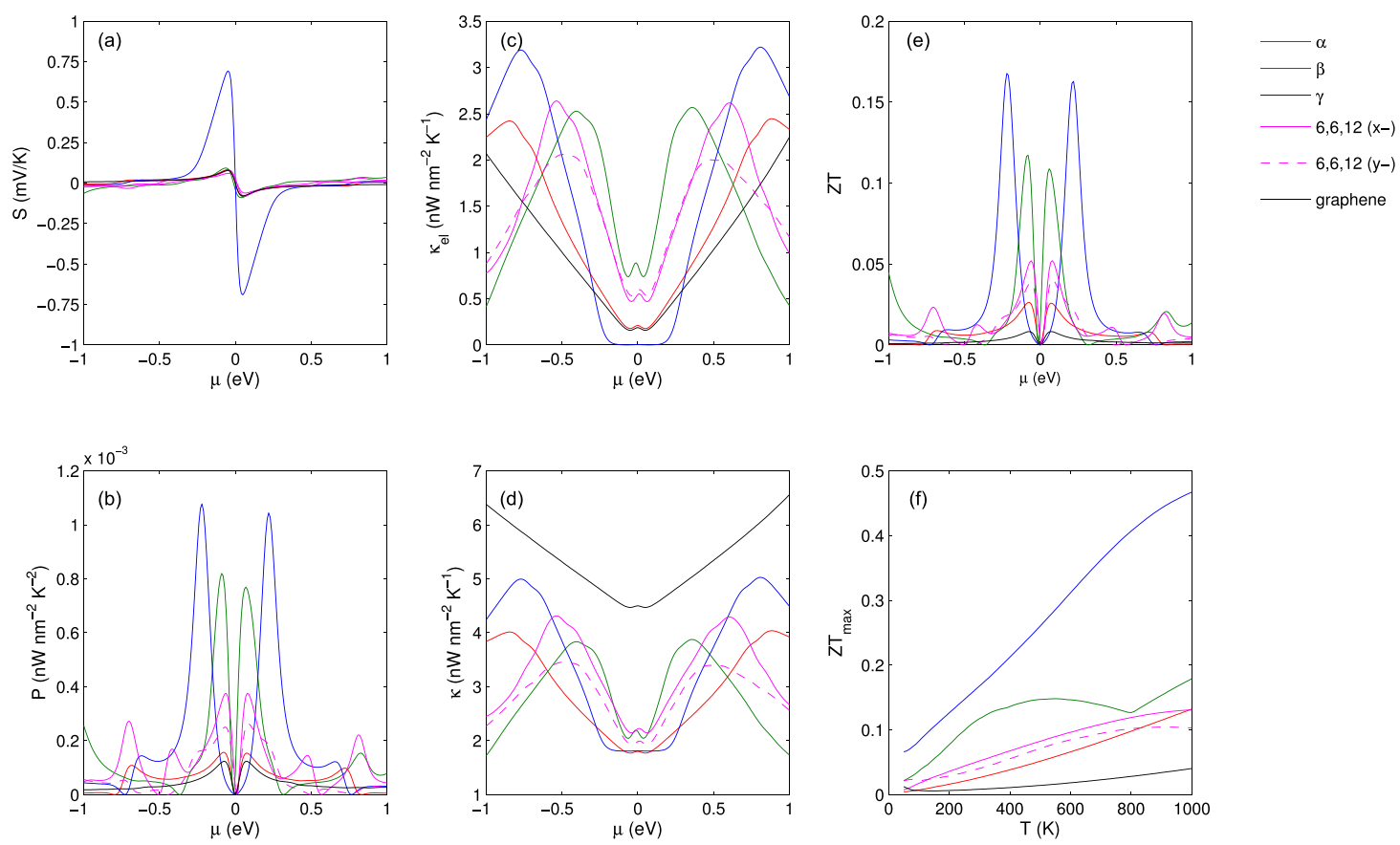

FIG. 4. Thermoelectric coefficients of graphynes and graphene. The Seebeck coefficient $S$ (a), the power factor $P=S^{2} G$ (b), electronic thermal conductance $\kappa_{\mathrm{el}} / A(\mathrm{c})$, total thermal conductance $\kappa / A(\mathrm{~d})$, and $Z T$ (e) are plotted at $T=300 \mathrm{~K}$ as functions of the chemical potential $\mu$. The maximum values of $Z T$ at different temperatures are also shown (f). 
range. $\alpha$ - and 6,6,12-graphynes are the least efficient graphynes thermoelectrically. A note about the band gap of $\gamma$ graphyne is in order here. It was reported that the band gap of $\gamma$-graphyne is $0.5 \mathrm{eV}$ larger when computed using HSE06 functional instead of PBE, while the effective masses of electrons and holes are not changed significantly by the choice of the functional. ${ }^{36}$ Our analysis shows that such an increase of the band gap does not change the thermoelectric efficiency at the valence and conduction band edges.

In conclusion, low energy electronic properties of $\alpha-, \beta$-, and 6,6,12-graphynes resemble massless Dirac fermions, while $\gamma$-graphyne has a $\sim 0.5 \mathrm{eV}$ band gap. Interestingly, graphene has the lower electrical transmittance than graphynes (it is higher than that of $\gamma$-graphyne only inside its gap). On the other hand, phononic thermal conductance of graphene is larger than that of the graphynes at temperatures higher than $60 \mathrm{~K}$. At $300 \mathrm{~K}, \kappa_{\mathrm{ph}} / A$ of graphene is about three times larger than graphynes. One of the reasons for superior $\kappa_{\mathrm{ph}} / A$ of graphene is the close packing, i.e., high areal density of bonds responsible for vibrational heat conduction. All Dirac materials studied in this work yield similar $S$ values at room temperature with a peak value of $0.09 \mathrm{mV} / \mathrm{K}$ close the CNP, but $\gamma$-graphyne, having a band gap, yields extremely high $S$ inside its gap and it is $0.15 \mathrm{mV} / \mathrm{K}$ at the band edges. Consequently, $\gamma$-graphyne is the most efficient thermoelectrically with $Z T$ values as high as 0.45 in the given temperature range, where graphene achieves less than 0.05 .

We would like to thank the ULAKBIM High Performance and Grid Computing Center for a sufficient time allocation for our projects. C.S. acknowledges the support from Scientific and Technological Research Council of Turkey (TUBITAK-113F096), Anadolu University (BAP1306F281 and BAP-1404F158), and Turkish Academy of Sciences (TUBA-GEBIP). H.S. acknowledges support from Scientific and Technological Research Council of Turkey (BIDEB-113C032) and Bilim Akademisi-the Science Academy, Turkey under the BAGEP program.

${ }^{1}$ K. Novoselov, A. Geim, S. Morozov, D. Jiang, Y. Zhang, S. Dubonos, I. Grigorieva, and A. Firsov, Science 306, 666 (2004).

${ }^{2}$ A. H. Castro Neto, F. Guinea, N. M. R. Peres, K. S. Novoselov, and A. K. Geim, Rev. Mod. Phys. 81, 109 (2009).

${ }^{3}$ K. Novoselov, A. Geim, S. Morozov, D. Jiang, M. Katsnelson, I. Grigorieva, S. Dubonos, and A. Firsov, Nature 438, 197 (2005).

${ }^{4}$ K. S. Novoselov, V. I. Fal'ko, L. Colombo, P. R. Gellert, M. G. Schwab, and K. Kim, Nature 490, 192 (2012).

${ }^{5}$ A. A. Balandin, S. Ghosh, W. Bao, I. Calizo, D. Teweldebrhan, F. Miao, and C. N. Lau, Nano Lett. 8, 902 (2008).

${ }^{6}$ W. Cai, A. L. Moore, Y. Zhu, X. Li, S. Chen, L. Shi, and R. S. Ruoff, Nano Lett. 10, 1645 (2010).

${ }^{7}$ J. H. Seol, I. Jo, A. L. Moore, L. Lindsay, Z. H. Aitken, M. T. Pettes, X. Li, Z. Yao, R. Huang, D. Broido, N. Mingo, R. S. Ruoff, and L. Shi, Science 328, 213 (2010).

${ }^{8}$ A. A. Balandin, Nat. Mater. 10, 569 (2011).

${ }^{9}$ F. Bonaccorso, Z. Sun, T. Hasan, and A. C. Ferrari, Nat. Photonics 4, 611 (2010).

${ }^{10}$ F. Schwierz, Nat. Nanotechnol. 5, 487 (2010).

${ }^{11}$ M. J. Allen, V. C. Tung, and R. B. Kaner, Chem. Rev. 110, 132 (2010).

${ }^{12}$ H. Sevinçli and M. Brandbyge, Appl. Phys. Lett. 105, 153108 (2014).

${ }^{13}$ S. Ghosh, W. Bao, D. L. Nika, S. Subrina, E. P. Pokatilov, C. N. Lau, and A. A. Balandin, Nat. Mater. 9, 555 (2010).

${ }^{14}$ S. Ghosh, I. Calizo, D. Teweldebrhan, E. P. Pokatilov, D. L. Nika, A. A. Balandin, W. Bao, F. Miao, and C. N. Lau, Appl. Phys. Lett. 92, 151911 (2008).
${ }^{15}$ S. Chen, Q. Wu, C. Mishra, J. Kang, H. Zhang, K. Cho, W. Cai, A. A. Balandin, and R. S. Ruoff, Nat. Mater. 11, 203 (2012).

${ }^{16}$ H. Sevincli, C. Sevik, T. Çă̆in, and G. Cuniberti, Sci. Rep. 3, 1228 (2013).

${ }^{17}$ J. Haskins, A. Kinaci, C. Sevik, H. Sevinçli, G. Cuniberti, and T. Çağin, ACS Nano 5, 3779 (2011).

${ }^{18}$ T. Gunst, T. Markussen, A. Jauho, and M. Brandbyge, Phys. Rev. B 84, 155449 (2011).

${ }^{19}$ D. Pacilé, J. C. Meyer, C. O. Girit, and A. Zettl, Appl. Phys. Lett. 92, 133107 (2008).

${ }^{20}$ R. Y. Tay, M. H. Griep, G. Mallick, S. H. Tsang, R. S. Singh, T. Tumlin, E. H. T. Teo, and S. P. Karna, Nano Lett. 14, 839 (2014).

${ }^{21}$ Q. H. Wang, K. Kalantar-Zadeh, A. Kis, J. N. Coleman, and M. S. Strano, Nat. Nanotechnol. 7, 699 (2012).

${ }^{22}$ L. Li, Y. Yu, G. J. Ye, Q. Ge, X. Ou, H. Wu, D. Feng, X. H. Chen, and Y. Zhang, Nat. Nanotechnol. 9, 372 (2014).

${ }^{23}$ A. N. Enyashin and A. L. Ivanovskii, Phys. Status Solidi B 248, 1879 (2011).

${ }^{24}$ M. T. Lusk and L. D. Carr, Carbon 47, 2226 (2009).

${ }^{25}$ N. K. Perkgoz and C. Sevik, Nanotechnology 25, 185701 (2014).

${ }^{26}$ M. M. Haley, Pure Appl. Chem. 80, 519 (2008).

${ }^{27}$ E. L. Spitler, C. A. Johnson II, and M. M. Haley, Chem. Rev. 106, 5344 (2006).

${ }^{28}$ T. Takeda, A. G. Fix, and M. M. Haley, Org. Lett. 12, 3824 (2010).

${ }^{29}$ X. Qian, Z. Ning, Y. Li, H. Liu, C. Ouyang, Q. Chen, and Y. Li, Dalton Trans. 41, 730 (2012).

${ }^{30}$ G. Li, Y. Li, H. Liu, Y. Guo, Y. Li, and D. Zhu, Chem. Commun. 46, 3256 (2010).

${ }^{31}$ S. W. Cranford and M. J. Buehler, Carbon 49, 4111 (2011).

${ }^{32}$ Y. Y. Zhang, Q. X. Pei, and C. M. Wang, Appl. Phys. Lett. 101, 081909 (2012).

${ }^{33}$ S. Ajori, R. Ansari, and M. Mirnezhad, Mater. Sci. Eng., A 561, 34 (2013).

${ }^{34}$ T. Ouyang, Y. Chen, L.-M. Liu, Y. Xie, X. Wei, and J. Zhong, Phys. Rev. B 85, 235436 (2012).

${ }^{35}$ X.-M. Wang, D.-C. Mo, and S.-S. Lu, J. Chem. Phys. 138, 204704 (2013).

${ }^{36}$ J. Kang, J. Li, F. Wu, S.-S. Li, and J.-B. Xia, J. Phys. Chem. C 115, 20466 (2011).

${ }^{37}$ J. Chen, J. Xi, D. Wang, and Z. Shuai, J. Phys. Chem. Lett. 4, 1443 (2013).

${ }^{38}$ D. Malko, C. Neiss, F. Viñes, and A. Görling, Phys. Rev. Lett. 108, 086804 (2012).

${ }^{39}$ V. N. Popov and P. Lambin, Phys. Rev. B 88, 075427 (2013).

${ }^{40}$ H. Lu and S.-D. Li, J. Mater. Chem. C 1, 3677 (2013).

${ }^{41}$ V. O. Ozcelik and S. Ciraci, J. Phys. Chem. C 117, 2175 (2013).

${ }^{42}$ Z. Liu, G. Yu, H. Yao, L. Liu, L. Jiang, and Y. Zheng, New J. Phys. 14, 113007 (2012).

${ }^{43}$ N. Narita, S. Nagai, S. Suzuki, and K. Nakao, Phys. Rev. B 58, 11009 (1998).

${ }^{44}$ M. Kondo, D. Nozaki, M. Tachibana, T. Yumura, and K. Yoshizawa, Chem. Phys. 312, 289 (2005).

${ }^{45}$ J. Zhou, K. Lv, Q. Wang, X. S. Chen, Q. Sun, and P. Jena, J. Chem. Phys 134, 174701 (2011).

${ }^{46}$ M. Schirber, Physics 5, 24 (2012).

${ }^{47}$ T. Ouyang, H. Xiao, Y. Xie, X. Wei, Y. Chen, and J. Zhong, J. Appl. Phys. 114, 073710 (2013).

${ }^{48}$ T. Ouyang and M. Hu, Nanotechnology 25, 245401 (2014).

${ }^{49}$ G. Kresse and J. Hafner, Phys. Rev. B 47, 558 (1993).

${ }^{50}$ G. Kresse and J. Furthmüller, Phys. Rev. B 54, 11169 (1996).

${ }^{51}$ P. Hohenberg and W. Kohn, Phys. Rev. 136, B864 (1964).

${ }^{52}$ W. Kohn and L. J. Sham, Phys. Rev. 140, A1133 (1965).

${ }^{53}$ R. M. Martin, Electronic Structure (Cambridge University Press, Cambridge, England, 2004).

${ }^{54}$ P. E. Blöchl, Phys. Rev. B 50, 17953 (1994).

${ }^{55}$ G. Kresse and D. Joubert, Phys. Rev. B 59, 1758 (1999).

${ }^{56}$ J. P. Perdew, K. Burke, and M. Ernzerhof, Phys. Rev. Lett. 77, 3865 (1996).

${ }^{57}$ H. J. Monkhorst and J. D. Pack, Phys. Rev. B 13, 5188 (1976).

${ }^{58}$ A. Togo, F. Oba, and I. Tanaka, Phys. Rev. B 78, 134106 (2008).

${ }^{59}$ S. Baroni, S. de Gironcoli, A. Dal Corso, and P. Giannozzi, Rev. Mod. Phys. 73, 515 (2001).

${ }^{60}$ L. G. C. Rego and G. Kirczenow, Phys. Rev. Lett. 81, 232 (1998).

${ }^{61}$ K. Esfarjani, M. Zebarjadi, and Y. Kawazoe, Phys. Rev. B 73, 085406 (2006).

${ }^{62}$ B. G. Kim and H. J. Choi, Phys. Rev. B 86, 115435 (2012).

${ }^{63}$ W. Wu, W. Guo, and X. C. Zeng, Nanoscale 5, 9264 (2013).

${ }^{64}$ H. Sevinçli and G. Cuniberti, Phys. Rev. B 81, 113401 (2010). 